\title{
Conscience de valeur et jouissance
}

Appendice IV in Edmund Husserl, Études concernant la structure de la conscience (1908-1914), volume 2, « Actes affectifs et donnée axiologique »

\section{Edmund Husserl}

Traducteur : Claudia Serban, Natalie Depraz et Maria Gyemant

\section{OpenEdition}

\section{Journals}

Édition électronique

URL : http://journals.openedition.org/alter/447

DOI : $10.4000 /$ alter.447

ISSN : 2558-7927

Éditeur :

Association ALTER, Archives Husserl (CNRS-UMR 8547)

Édition imprimée

Date de publication : 1 décembre 2016

Pagination : 249-255

ISBN : 978-2-9550449-2-6

ISSN : 1249-8947

\section{Référence électronique}

Edmund Husserl, « Conscience de valeur et jouissance », Alter [En ligne], 24 | 2016, mis en ligne le 01

décembre 2017, consulté le 19 avril 2019. URL : http://journals.openedition.org/alter/447 ; DOI :

10.4000/alter.447 


\section{APPENDICE IV $<$ CONSCIENCE DE VALEUR ET JOUISSANCE $>1$}

[A VI 8 I/52 a "I"] Vivre dans l'acte : en tant que s'accomplir, s'adonner aux actes affectifs en tant que libre adonnation (Hingegebenheit). Abstention: abstention du jugement, de la joie (abstention dans la jouissance), abstention de la volonté. Valeur et jouissance de la chose de valeur.

Je peux trouver une chose réjouissante sans m'en réjouir effectivevement. Je suis peut-être trop hébété pour m'en réjouir, trop émoussé. Je me fâche de ne pas pouvoir m'en réjouir, car je suis pourtant persuadé que cela est beau, qu'il en est effectivement ainsi ou que l'événement s'est produit. Je peux en effet « reconnaître » que cela est réjouissant.

Comment peut-on expliquer cela ? Il peut y avoir conflit de motifs. Par exemple, je peux reconnaître que le fait que la Russie a subi une défaite est une bonne chose. Mais j'ai des titres de rente russes et je ressens les dommages de la chute du marché, qui est une suite de la défaite. Je ne peux donc pas me réjouir de la victoire des Japonais que je trouve réjouissante par ailleurs ${ }^{2}$. Ou quelqu'un me fait part du fait qu'un de mes souhaits de longue date s'est réalisé. Je ne suis pourtant pas dans l'attitude de m'en réjouir. Je sais que l'état de choses qui s'est produit là est réjouissant, que je devrais me réjouir, mais je ne me réjouis pas.

Ou bien je suis assoupi, j'applique toutes mes pensées et mes sentiments à tout autre chose. Et pourtant je comprends tout de suite qu'il s'agit d'un événement réjouissant, même avant de m’en réjouir effectivement. Dans de tels cas, j'ai dans un face à face ce qui

${ }^{1}$ 1909. - Nota bene ! Très important.

${ }^{2}$ Husserl se réfère ici vraisemblablement à la guerre russo-japonaise (1904-1905), qui a trouvé une fin avec la signature du traité de paix de Portsmouth le 5 septembre 1905. Note de l'éditeur. 
est conscient, pensé et jugé comme réjouissant et [52 b] la joie effective.

Ne puis-je pas aussi reconnaître dans l'évidence que quelque chose est réjouissant, sans aucune joie effective ? Selon toute apparence, il y a un parallélisme en général: je peux "voir» qu'une image est "attirante", sans pour autant être capable sur le moment de $\mathrm{m}^{\prime}$ adonner à l'attrait. Je peux « voir » une valeur esthétique sans pour autant jouir d'elle. Je peux sentir la saveur d'une nourriture sans en «jouir». Je tiens quelque chose pour souhaitable, pour digne d'être désiré, et pourtant je ne le souhaite pas, ne le désire pas. Je tiens quelque chose pour bon, pour digne d'être voulu, sans pour autant le vouloir. Je ne me décide pas d'un point de vue pratique pour cela : [pourtant] tout parle en sa faveur et rien contre lui. Je ne pense pas non plus à l'effort qui serait requis pour mettre en œuvre ce qui est voulu. Je suis juste purement passif. Je ne fais rien.

Peut-on aussi dégager des exemples à partir de la sphère du jugement ? Je tiens vraiment une chose pour juste, mais je ne vais pas me l'avouer. Je dis autre chose. Je garde mon opinion de parti. Non seulement je l'exprime à travers des mots, mais j'y "crois » aussi. Je sens le poids supérieur des arguments en faveur d'une doctrine, mais « je ne la (ou le) laisse pas valoir». D'autres convictions s'opposent à elle, convictions que je veux garder, auxquelles je tiens avec tout mon cœur, que je préfère de tout mon cœur. Je cherche des raisons, éventuellement des raisons apparentes, qui pourraient neutraliser ce poids supérieur, ne serait-ce qu'en apparence. Ce qui s'impose comme vrai n'est pas accepté par moi comme vrai, mais je prends position à son égard en le rejetant. Or puis-je effectivement voir que $S$ est $P$ et pourtant « prendre position » contre cela?

[A VI 8 I/53 a "II"] On pourrait aussi renvoyer au phénomène de $l^{\prime}$ abstention de juger. Je vois que $S$ est $P$. « $S$ est $P$ » se tient devant moi! Je m'abstiens pourtant du jugement. Je ne veux pas, maintenant, "prendre position" pour, mais pas non plus contre. Je prends la position de l'abstention de juger.

Ne puis-je pas $\mathrm{m}^{\prime}$ «abstenir » aussi de la joie? Je vois que quelque chose est réjouissant, la chose se tient là en tant que réjouissante : je ne veux pourtant pas prendre position à son égard sur le mode de la joie active. Et même si je m'en réjouis déjà, je peux toujours m'abstenir de la joie active.

Puis-je semblablement m'abstenir d'un souhait, d'un désir, même si la situation apparaît comme souhaitable (digne d'être souhaitée), comme agréable ? On peut s'adonner à un souhait ou à un désir, on 
parle alors d'un souhait vif, brûlant, et «plus » je m'y adonne, plus il gagne de force sur moi et plus il devient vif, et ainsi de suite. Je peux m'adonner à une conviction. En cohésion avec cela se tient le fait qu'une conviction peut être plus ou moins vive.

Avons-nous quelque chose d'analogue dans le cas du vouloir? Vivacité du vouloir, puissance, «énergie »du vouloir ? S'agit-il ici de moments immanents de l'acte ou bien de caractères du contexte ? Je vis dans le vouloir, le vouloir gagne dans la vie de conscience une position insigne, dominante, inhibitrice, etc., qui exclut d'autres actes, qui domine l'écoulement des actes, etc.

Est-ce l'inverse quand il s'agit de la joie, pour revenir à elle ? [53 b] En est-il ainsi : je tiens quelque chose pour réjouissant, non seulement "théoriquement», à la manière d'un savoir du fait qu'il est réjouissant et éventuellement aussi du fait qu'il réclame de la joie, etc., mais je le «vois » comme réjouissant? Un acte de la joie appartient aussi à cela ${ }^{3}$.

L'on pourrait dire à présent qu'il y a différentes attitudes : 1) Je me réjouis de l'événement. 2) J'ap<erçois> l'événement comme réjouissant. «Je perçois en lui la réjouissance, la beauté, la coloration rosée. $»^{4} \mathrm{~L}^{\prime}$ acte détient là une fonction différente. Mais dans le deuxième cas aussi, un se-réjouir-de est certainement présent.

Mais dans le mode de l'accomplissement d'acte il y a encore une autre différence. Je me réjouis, non seulement dans la mesure où j'ai été contraint de constater ( « percevoir») la chose réjouissante, je m'adonne aussi à la joie. Je vis dans la jouissance, d'une manière qui est ou n'est pas totalement exclusive. Je me réjouis, mais entre temps je fais aussi attention à autre chose, je m'occupe aussi d'autre chose, etc. Finalement il se peut que je «n'aie pas le temps de me réjouir». Je jette des regards rapides sur la chose, des regards de joie, mais le centre de ma vie doit résider ailleurs. Je ne peux pas, dans les circonstances actuelles de vie, m'adonner à une telle joie. Mon intérêt, ma force appartient à autre chose. Détendu par la jouissance, je dois pourtant demeurer en tension, maintenir mon énergie rassemblée, etc.

$\mathrm{Ou}$ bien je jouis certes, mais avec retenue: je dois toujours demeurer prêt pour ce que peut apporter l'heure. De même que le soldat qui veille n'est pas libre dans la jouissance et ne s'y adonne pas librement, mais s'attend constamment à l'assaut de l'ennemi. L'acte de la joie est là, mais d'une manière différente. Il n'est ni énergie libre,

\footnotetext{
${ }^{3}$ Mais comment? Il faut réfléchir à cela.

${ }^{4}$ C'est-à-dire: j'accomplis un «acte d'entendement», une «représentation », [une] position théorique, un jugement.
} 
ni énergie inhibée, et l'inhibition (Hemmung) peut être inhibition émanant d'autres actes de joie, etc., ou d'une volonté inhibante, d'une «abstention». Un repoussoir arbitraire par opposition à l'acte qui se déploie librement à l'infini, par opposition au fait de s'adonner sans bornes (qui peut être arbitraire ou non). Il s'agit là bien sûr de différences phénoménologiques.

[A VI 8 I/54 a "III"] Il faut remarquer à ce propos: lorsque je " vois » quelque chose de réjouissant en tant que tel, le moment du [se-]réjouir n'est pas caractérisé comme un se-réjouir au sens courant. Très courantes sont les fluctuations: Je tiens quelque chose pour réjouissant, je sais qu'il l'est, je m'adonne alors à la joie (je m'en réjouis maintenant). Ou bien je $\mathrm{m}^{\prime}$ en réjouis, je constate la réjouissance, je vois l'objet dans son être-réjouissant, je m'adonne alors doublement à la joie, lorsque je vois cela, etc.

Quand je $\mathrm{m}^{\prime}[\mathrm{y}]$ adonne, ce qui est réjouissant se tient déjà là en tant que réjouissant. Le beau se tient là en tant que beau. Dans l'attitude objective que j'ai de prime abord, le beau ressortit objectivement. Je sens l'attrait qu'il exerce, et pourtant je ne vis pas dans le sentiment, mais dans la conscience d'objectité, et cela implique une conscience d'appréhension $[\mathrm{du}]$ « beau ». Quand je m'[y] adonne, «je $\mathrm{m}^{\prime}$ adonne à la beauté », et d'une certaine manière «je m'adonne à la joie que suscite la beauté, à la jouissance de la beauté ». Le beau se tient déjà là <en tant que> beau, et je $\mathrm{m}^{\prime}[\mathrm{y}]$ tourne dans le plaisir de la beauté. L'objet beau $\underline{m}^{\prime}$ invite avec sa beauté à y prendre plaisir, à vivre dans la joie jouissante (à me jeter en un autre sens dans les bras de la jouissance, etc.).

À ce propos il est inutile de dire qu'il y a là une variété du sentiment : un sentiment qui appartient à la constitution de la beauté à même l'objet et un autre qui appartient au plaisir. (Ainsi, pour invoquer un cas parallèle quoique déjà différent, la même sensation tactile [renvoie] dans un cas à l'aspérité de l'objet, et selon l'autre direction d'appréhension, à la sensation d'aspérité dans la main, appartenant au sujet.)

[54 b] Le caractère objectif, le caractère de la beauté, de la valeur, etc., ne change pas en fonction de l'absence de bornes du «fait de s'adonner ", c'est-à-dire en fonction de sa limitation et de l'abstention. La joie peut devenir de plus en plus vive aussi bien lorsqu'on s'y adonne librement que proportionnellement à la mise hors circuit des moments inhibiteurs. Mais c'est là quelque chose qui relève du comportement actif et qui ne change rien à la valeur de la chose, telle qu'elle est naturellement consciente en tant que valeur. C'est là pourtant un terrain pour des recherches. 
Bien sûr, la valeur relative change à chaque fois qu'à sa suite des valeurs comparativement plus hautes ou plus basses sont introduites dans le [même] milieu ou, plus généralement, font leur entrée et se font entendre dans le champ visuel de la conscience. Mais lorsque, par exemple, je concentre mon attention sur une nourriture et sur sa saveur, il est propice de la saisir en la détachant du reste afin de la déterminer dans sa spécificité avec évidence et clarté. Et si je $\mathrm{m}^{\prime}$ adonne librement à la jouissance, celle-ci sera à présent plus vive que lorsque j'étais déconcentré ou dans d'autres cas semblables. Mais la jouissance plus vive ne rend pas la saveur plus intense : celle-ci vaut pour moi comme inchangée, elle appartient à l'objet. Je dis aussi qu'elle parvient mieux à la validité, à son droit, etc. Mais cela implique la même chose : l'être-objectif de la propriété de valeur.

Nous aurions aussi à dire ceci : il se peut qu'une puissance désirante devienne de plus en plus puissante et que ce que je désire, sa valeur, soit surestimé en fonction de l'intensité du désir. Des illusions quant aux degrés de valeur sont possibles et sont conditionnées aussi par l'intensité avec laquelle on s'adonne à la jouissance, par l'intensité du sentiment de l'affect auquel on s'adonne. Et pourtant nous parlons d'illusions. Il appartient à la « nature » de ces rapports entre valeur et jouissance que la valeur soit quelque chose d'objectif et qu'elle ne soit pas déterminée par le degré changeant de la jouissance. Mais ce n'est là qu'un début!

[A VI 8 I/55 a "IV"] Compléments : Dans la sphère du jugement, et de prime abord dans le cas des jugements au sens plus étroit d'actes énonciatifs, affirmatifs ou de croyance, nous distinguons le caractère du jugement au sens logique, voire phanséologique, grâce auquel l'affirmation est bien une affirmation, S est P !, [grâce auquel] la croyance croit bien cela et ainsi de suite. Par opposition à cela, nous distinguons d'autre part l'être-convaincu plus ou moins vif, la conscience de la malhonnêteté, de la partialité, qui a réprimé les motifs contraires ou ne les a pas laissés apparaître, etc. Tout cela se rapporte aux modes subjectifs de l'accomplissement du «même jugement ».

Au même registre appartient aussi un sens de l'expression " prise de position» souvent accepté (en vertu duquel le mot ${ }^{5} \underline{\text { n}}^{\prime}$ est pas

\footnotetext{
$5<$ Prise de position $>=$ comme prise de parti. Prendre parti peut vouloir dire consentir ; je peux pourtant aussi prendre parti, expliquer que je suis d'accord, me positionner du côté de celui qui juge, prendre parti pour son avis, m'engager pour cela par le sentiment. Je peux pourtant aussi être intéressé à un contenu de jugement qui s'impose à moi, être intéressé, avoir un intérêt au fait de l'adopter, de le laisser valoir, de le juger.
} 
utilisable pour la qualité d'acte ou l'intention d'acte), selon lequel nous souscrivons à une vue, nous nous positionnons à ses côtés, ce qui confère au jugement un mode qui ne l'altère pas en tant que jugement (en tant que croyance que cela soit ainsi ou ainsi, eu égard à cette généralité qui justifie globalement le fait de parler de la même croyance, du même jugement).

Et le mode de cette prise de position est alors encore différent, dans la mesure où elle se relie par là à une prise de parti honnête ou malhonnête ou autre. Et le contraire est de ne-pas-prendre-position (abstention) ou de prendre-position-contre comme conscience-denégation.

[55 b] Il en va semblablement pour la supposition, le tenir-pourprobable, le douter (doute malhonnête, émanant de l'esprit de parti) et ainsi de suite. Nous voyons encore quelque chose d'analogue dans la sphère de l'affectivité.

Il se pose à présent certes la question de savoir si dans la sphère du jugement nous n'avons pas simplement affaire à de simples caractères affectifs, des sentiments, des tendances qui produisent les différences. En partie, tout cela joue naturellement aussi. Mais toutefois pas autrement que dans le cas des actes affectifs eux-mêmes ${ }^{6}$, avec lesquels des tendances peuvent se lier et sont souvent liées, tendances qui, en s'épanouissant, déterminent elles-mêmes le caractère de l'acte affectif, tandis que nous y vivons jusqu'au bout ou pas, etc.

Dans tous les cas nous avons une sphère de motivations qui traversent tous les types d'actes (types d'impression).

1) Nous avons en général à distinguer ce qui appartient à la "qualité d'acte » correspondante, au mode de l'intention, qui est la seule à être pertinente pour la question de droit (à côté de la matière), de sorte que nous jugeons en général fondamental le concept de $l^{\prime}$ " essence intentionnelle » comme produit de la matière intentionnelle et de la qualité intentionnelle ; 2) et ce qui appartient au mode de $l^{\prime}$ «accomplissement» de l'intention et qui entraîne avec soi des différences relatives à l'intensité.

L'affirmation, le jugement ne connaissent pas de différence d'intensité : dans leur essence intentionnelle. Mais pour ce qui a trait aux actes affectifs, nous avons même là des différences d'intensité, et à côté de cela encore des différences relatives à l'intensité dans le mode de l'accomplissement, qui sont de telle sorte qu'elles contribuent au

\footnotetext{
${ }^{6}$ Pas autrement? Je m'adonne à un souhait ou à une joie, je vis pleinement en eux. Pas au même sens dans le jugement. Les motifs de s'adonner sont à distinguer du fait de s'adonner lui-même et de son intensité changeante.
} 
fait que nous pouvons commettre des méprises dans l'attribution de valeur ${ }^{7}$.

Traduit par Claudia Serban

en collaboration avec Natalie Depraz et Maria Gyemant ${ }^{8}$

${ }^{7}$ En marge du dernier paragraphe Husserl a ajouté plus tard un point d'interrogation. Note de l'éditeur.

${ }^{8}$ Nous remercions les éditeurs du volume allemand, Ü. Melle et Th. Vongehr, ainsi que les Archives-Husserl, d'avoir autorisé la parution de ce texte. 